\title{
Hyperactivation of retina by light in mice leads to photoreceptor cell death mediated by VEGF and retinal pigment epithelium permeability
}

\author{
M Cachafeiro ${ }^{1,6}$, A-P Bemelmans ${ }^{\star, 1,2,6,7}$, M Samardzija $^{3}$, T Afanasieva ${ }^{4}$, J-A Pournaras ${ }^{5}$, C Grimm $^{3}$, C Kostic $^{1}$, S Philippe $^{1}$, A Wenzel $^{3}$ \\ and Y Arsenijevic ${ }^{* 1}$
}

Light toxicity is suspected to enhance certain retinal degenerative processes such as age-related macular degeneration. Death of photoreceptors can be induced by their exposure to the visible light, and although cellular processes within photoreceptors have been characterized extensively, the role of the retinal pigment epithelium (RPE) in this model is less well understood. We demonstrate that exposition to intense light causes the immediate breakdown of the outer blood-retinal barrier (BRB). In a molecular level, we observed the slackening of adherens junctions tying up the RPE and massive leakage of albumin into the neural retina. Retinal pigment epithelial cells normally secrete vascular endothelial growth factor (VEGF) at their basolateral side; light damage in contrast leads to VEGF increase on the apical side - that is, in the neuroretina. Blocking VEGF, by means of lentiviral gene transfer to express an anti-VEGF antibody in RPE cells, inhibits outer BRB breakdown and retinal degeneration, as illustrated by functional, behavioral and morphometric analysis. Our data show that exposure to high levels of visible light induces hyperpermeability of the RPE, likely involving VEGF signaling. The resulting retinal edema contributes to irreversible damage to photoreceptors. These data suggest that anti-VEGF compounds are of therapeutic interest when the outer BRB is altered by retinal stresses.

Cell Death and Disease (2013) 4, e781; doi:10.1038/cddis.2013.303; published online 29 August 2013

Subject Category: Neuroscience

Age-related macular degeneration (AMD), a main reason for visual impairment in the elderly, ${ }^{1}$ is linked to pathological changes involving the retinal pigment epithelium (RPE), a mono-stratified cell layer with multiple functions in relation to photoreceptor physiology. The RPE constitutes the outer blood-retinal barrier (BRB), controls fluid and metabolic exchange between the retina and the choriocapillaris, and participate in several functions linked to photoreceptor physiology. In addition, RPE cells release factors controlling neuronal survival as well as angiogenesis, such as pigment epithelium-derived factor (PEDF), which promote photoreceptor survival and have anti-angiogenic action on the choriocapillaris. ${ }^{2,3}$ The RPE secretes PEDF at the apical side - that is, towards the neural retina - and, in contrast, the angiogenic factor vascular endothelial growth factor (VEGF) is secreted at the basolateral side to maintain the fenestration of the choriocapillaris. ${ }^{4-6}$ As a consequence, unbalanced expression of PEDF and VEGF appears to be involved in major human pathologies such as choroidal neovascularization (CNV) in $\mathrm{AMD}^{7}$ or diabetic retinopathy. ${ }^{8}$ Anti-VEGF treatments have thus become the therapeutic option of choice for the management of the wet-form of AMD involving CNV.

In contrast to in vitro data, ${ }^{9,10}$ few studies focused on RPE cell alteration after in vivo stress. Genetic studies revealed important mechanisms involved in the process of RPE alteration and photoreceptor death, such as complement factor $\mathrm{H}$ loss of function or ApolipoproteinE4; however, the

${ }^{1}$ Unit of Gene Therapy \& Stem Cell Biology, Jules-Gonin Eye Hospital, University of Lausanne, Lausanne, Switzerland; ${ }^{2}$ Institut de la Vision, UPMC Univ Paris 06, UM80, INSERM, U968, CNRS, UMR_7210, Paris, France; ${ }^{3}$ Laboratory for Retinal Cell Biology, Department of Ophthalmology, University of Zurich, Zurich, Switzerland; ${ }^{4}$ Division of Cancer Research, Department of Pathology, University Hospital, Zürich, Switzerland and ${ }^{5}$ Vitreoretinal Surgical Unit, Jules-Gonin Eye Hospital, University of Lausanne, Zurich, Switzerland

*Corresponding author: Y Arsenijevic, Unit of Gene Therapy \& Stem Cell Biology, Hôpital ophtalmique Jules-Gonin, Avenue de France 15, Lausanne 1004, Switzerland. Tel: +41 02162682 60; Fax: +41 02162688 88; E-mail: yvan.arsenijevic@fa2.ch

or A-P Bemelmans, Molecular Imaging Research Center, URA2210 CEA/CNRS, 18 Route du Panorama, Fontenay-Aux-Roses 92260 , France. Tel: +33 0146549629 ; Fax: +33 1465491 16; E-mail: alexis.bemelmans@cea.fr

${ }^{6}$ These authors contributed equally to this work.

${ }^{7}$ Present address: Molecular Imaging Research Center, URA2210 CEA/CNRS, 18 Route du Panorama, 92260 Fontenay-Aux-Roses, France.

Keywords: neurodegeneration; photoreceptor; VEGF; retina; gene therapy

Abbreviations: A2E, N-retinylidene-N-retinylethanolamine; AMD, Age-related macular degeneration; AP-1, activator protein-1; BRB, blood-retinal barrier; BSA, bovine serum albumin; cDNA, complementary deoxyribonucleic acid; CNV, choroidal neovascularization; cpd, cycles per degree; DAPI, 4',6'-diamidino-2-phenylindole; EDTA, Ethylenediaminetetraacetic acid; EFS, elongation factor1-alpha short promoter; ELISA, enzyme-linked immunosorbent assay; ERG, electroretinogram; GFP, green fluorescent protein; HIV-1, human immunodeficiency virus-1; IRES, internal ribosome entry site; LD, light-damage; MAPK, mitogen-activated protein kinase; NGS, normal goat serum; ONL, outer nuclear layer; OR, optokinetic reflex; OS, outer segments; PBS, phosphate-buffered saline; PEDF, pigment epithelium-derived factor; PFA, paraformaldehyde; PI3, Phosphatydil-inositol-3-phosphate; ROS, reactive oxygen species; RPE, retinal pigment epithelium; SWL, short-wave length; TBS-T, Trisbuffered saline containing $0.1 \%$ Tween-20; VEGF, vascular endothelial growth factor; VEGFR-2, vascular endothelial growth factor receptor-2; VSV-G, vesicular stomatitis virus G-protein

Received 28.3.13; revised 22.5.13; accepted 17.6.13; Edited by D Bano 
mechanisms initiating deleterious effects of these gene variants are unknown. ${ }^{11}$ Moreover, the role of environmental detrimental stimuli is poorly understood and is mainly based on hypotheses generated from human retina samples (reviewed by Bird ${ }^{12}$ ). On the other hand, among environmental factors that may impair photoreceptor survival, light toxicity has been intensely investigated. Indeed, several studies have shown that exposure to high-intensity of light induces photoreceptor loss (light-damage model or LD) by several mechanisms that can be specific to the LD model ${ }^{13}$ and by some other mechanisms that are common to other inherited retinal dystrophies. ${ }^{14,15}$ Interestingly, different studies showed that after LD the retina presents some cardinal features of AMD. ${ }^{16-19}$ For instance, LD leads to the accumulation of reactive oxygen species and generation of toxic metabolites such as $\mathrm{N}$-retinylidene-N-retinylethanolamine (A2E), partially degraded proteins and lipid-protein adducts. ${ }^{20}$ Moreover, high levels of environmental light have been implicated in the accumulation of drusen, ${ }^{21}$ and photooxidized A2E appears to activate the complement system, ${ }^{22}$ suggesting that RPE dysfunction may contribute to the degeneration of photoreceptors occurring after LD. Interestingly, a recent study shows that an acute stress caused by chemical oxidative damage leads to RPE dysfunction and photoreceptor stress. ${ }^{23}$ However, no direct evidence of the role of RPE on photoreceptor death after physiological stress, such as high-light exposure, has been provided so far.

In the present study, we aimed to determine whether the RPE is also affected during the LD process and to evaluate the degree of RPE involvement in the process of photoreceptor death. We show the crucial contribution of RPE in the induction of photoreceptor death process after LD, and that VEGF drives outer BRB breakdown constituting an additional mechanism of retinal fluid accumulation in these conditions.

\section{Results}

VEGF release and RPE permeability are the early events in the light-damage model. In the LD model, and in our experimental conditions, a 1-h exposure to 5000 lux induced dramatic photoreceptor degeneration (Figure 1). Photoreceptor cell death was evidenced on cresyl violet-stained sections (Figures 1a-d). Cell death occurred through an apoptotic mechanism leading to an increase in the nucleosome-free fraction (Figure 1e). To decipher the role of the RPE in this model, we studied the status of adherens- and tight-junction proteins unifying this mono-layered epithelium and making the outer BRB. ${ }^{24-28}$ On control flat-mounted RPE, zonula-occludens-1 (ZO-1), a protein involved in tight junction, and proteins building adherens junctions, such as beta-catenin and $\mathrm{N}$-cadherin, precisely delineate the contour of RPE cells, revealing their hexagonal shape (Figures $2 a$ and $c-e$ ). Twenty-four hours after LD, these three markers have completely left their locations associated with the plasma membrane and are translocated into the cell cytoplasm (Figures 2b, $d$ and f), demonstrating the loss of RPE cell-cell connection integrity. To determine whether the disruption of RPE junction markers resulted in changes of the $\mathrm{BRB}$ permeability, extravascular plasma leakage was evaluated by immunostaining of serum albumin on retinal cryostat sections sampled at different times after LD. In control eyes, albumin was found only in the choriocapillaris layer and in the retinal vessels located at the inner side of the retina (Figures $2 \mathrm{~g}$ and $\mathrm{h}$ ). However, albumin staining was clearly visible in the outer segment (OS) layer and massively spread toward the inner layers at 24, 36 and 48-h post $L D$, indicating a plasma leakage originating from the outer BRB (Figures 2i-n). Albumin leakage was only observed in the central retina affected by LD and in the sites where the photoreceptors were degenerating, but not in the peripheral retina, which was preserved after light exposure. These observations showed that after light damage, large plasmatic proteins can pass the outer BRB, revealing that RPE cannot maintain fluid and protein homeostasis in this circumstance and suggest that this leakage contributes to retinal degeneration.

Similar to blood capillaries, RPE permeability closely depends on the availability of pro- and anti-angiogenic factors such as VEGF and PEDF. ${ }^{9}$ To determine whether these factors could be involved in the RPE permeability occurring in the LD model, we quantified the expression
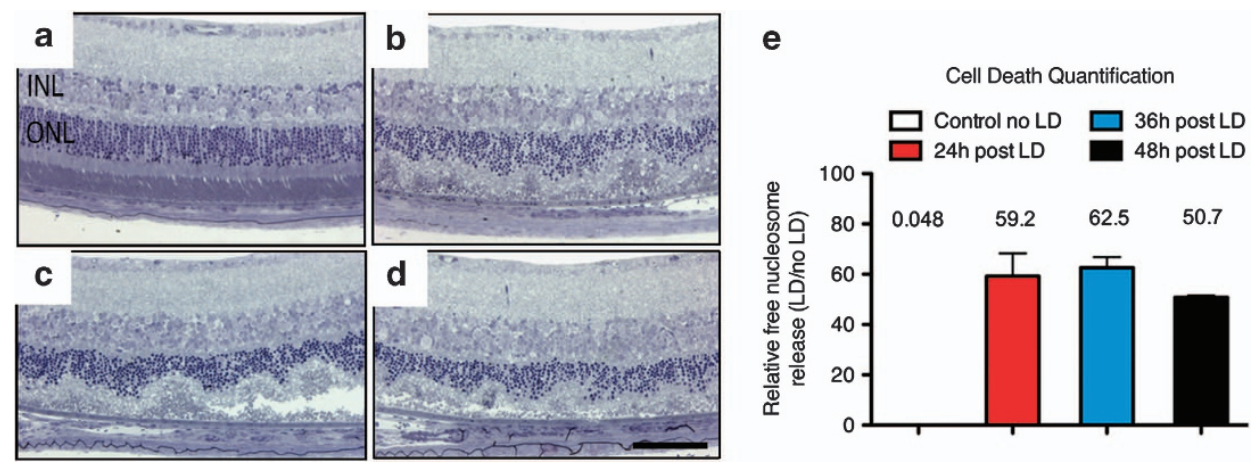

Figure 1 Light-induced photoreceptor degeneration by apoptosis. Balb/c mice were exposed to 5000 lux of white light during $1 \mathrm{~h}$. Compared with retina unexposed to light (a), cresyl violet staining of mice, respectively, analyzed 24,36 and $48 \mathrm{~h}$ after LD (b-d) showed that LD-induced photoreceptor cell death progressed with time. (e) Cytoplasmic-free nucleosomes in the retina were quantified using an ELISA-based cell death detection kit at the different time points indicated. One-way ANOVA (Dunnett's post hoc test) analysis revealed that the number of free nucleosomes released after LD were significantly higher 24,36 and $48 \mathrm{~h}$ after the lesion compared with animals unexposed to $L D(P<0.001$ for all time points studied). Each bar represents the mean value \pm S.D. ( $n=6$ per group). ONL: outer nuclear layer, INL: inner nuclear layer, scale bar: $50 \mu \mathrm{m}$ 


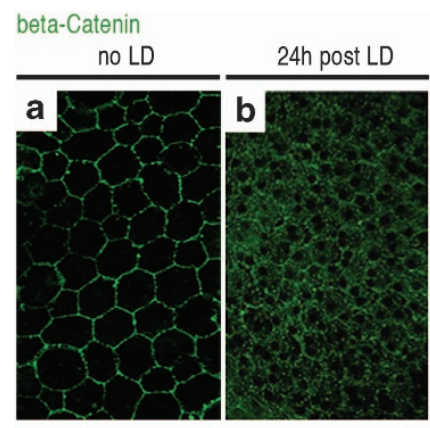

Z01 no LD
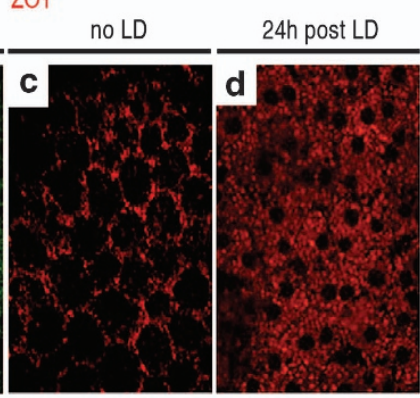

$\mathrm{N}$-Cadherin no LD 24h post LD

no LD
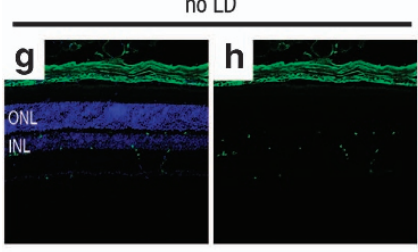

36h post LD

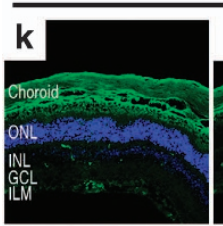

Albumin/DAPI

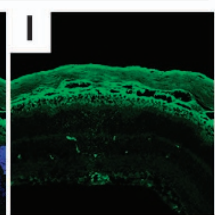

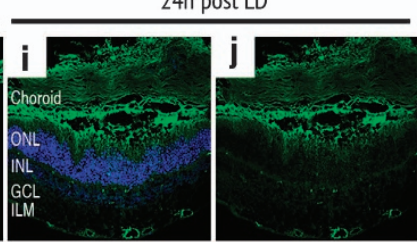

48h post LD

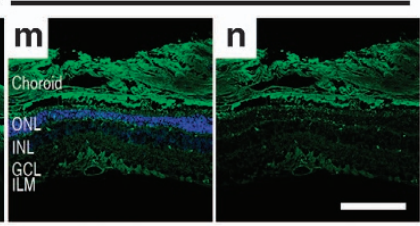

-
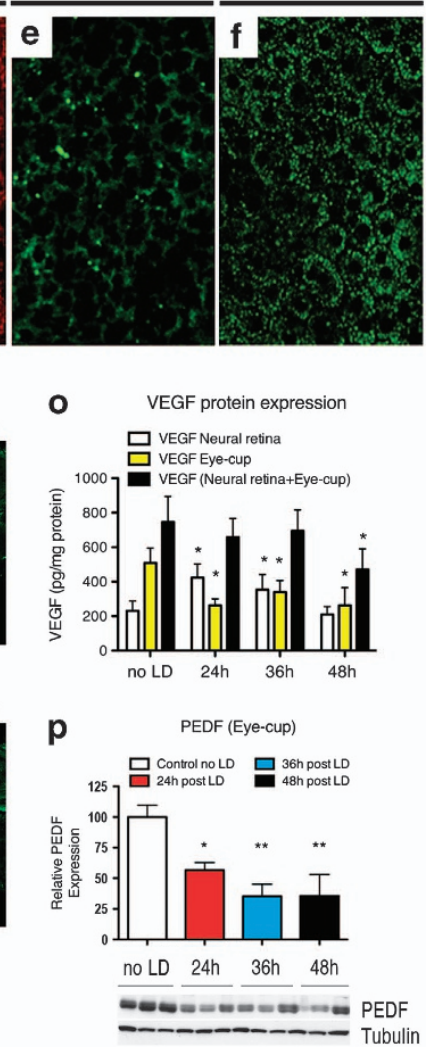

Figure 2 VEGF-triggered RPE permeability is an early event in the light-damage model. Confocal analysis of tight- and adherens-junction markers performed on flat-mounted RPE revealed that RPE permeability is an early event in the LD model. Whereas the beta-catenin expression delineates the contour of RPE cells revealing their hexagonal shape on control unexposed to light (a), the expression of this marker was localized in the cytoplasm of RPE cells $24 \mathrm{~h}$ after LD (b). Similar results were observed concerning the expression of ZO-1 and N-cadherin markers. Normally located in the contour of RPE cells on control unexposed to light (c-e), both of these markers translocate in the RPE cell cytoplasm $24 \mathrm{~h}$ after LD $(\mathbf{d}-\mathbf{f})(n=6$ per group - magnification $\times 400)$. Immunostaining of serum albumin, normally found in the choriocapillaris and in the retinal vessels located at the inner side of the retina of control unexposed to light ( $\mathbf{g}$ and $\mathbf{h}$ ), showed pronounced extravascular albumin leakage 24 (i and $\mathbf{j})$, 36 ( $\mathbf{k}$ and $\mathbf{I}$ ) and $48 \mathrm{~h}(\mathbf{m}$ and $\mathbf{n}$ ) after light exposure. After LD, albumin was prominent in the RPE and in the outer nuclear layer (ONL). Albumin positivity was also found, to a lesser extent, in the outer plexiform layer (OPL), in the inner nuclear layer (INL), in the plexiform layer (IPL), in the ganglion cell layer (GCL) and, finally, in the inner limiting membrane (ILM), suggesting that plasma leakage originates from the outer BRB ( $n=6$ per group-magnification $\times 400$, scale bar: $100 \mu \mathrm{m}$ in (g-n)-Green: albumin and blue: DAPI). (o) ELISA quantification shows that VEGF level was 1.8-fold and 1.5-fold increased, respectively, 24 and $36 \mathrm{~h}$ after LD compared with control unexposed to light in the neural retina (control no LD: $231.6 \pm 56.3 \mathrm{pg} / \mathrm{mg}$ protein; $24-\mathrm{h}$ post LD: $423.5 \pm 79.3 \mathrm{pg} / \mathrm{mg}$ protein; 36 -hour after LD: $353.8 \pm 87.7 \mathrm{pg} / \mathrm{mg}$ protein and 48-h post LD: $209.7 \pm 45.3 \mathrm{pg} / \mathrm{mg}$ protein). Contrariwise, in the eye-cup, VEGF content was significantly decreased 24,36 and $48 \mathrm{~h}$ after LD compared with unexposed controls (control no LD: $508.9 \pm 96.2 \mathrm{pg} / \mathrm{mg}$ protein; $24-\mathrm{h}$ post LD: $262.9 \pm 37.3 \mathrm{pg} / \mathrm{mg}$ protein; $36-\mathrm{h}$ post LD: $340.7 \pm 88.8 \mathrm{pg} / \mathrm{mg}$ protein and $48-\mathrm{h}$ post LD: $262.7 \pm 132.4 \mathrm{pg} / \mathrm{mg}$ protein). No obvious difference was observed concerning VEGF content in the whole eye, except at $48 \mathrm{~h}$ after LD. At this point, VEGF was 1.5 -fold decreased compared with the control. Each bar represents the mean value \pm S.D. ( $n=6$ per group). Statistical analyses: one-way ANOVA and Dunnett's post hoc test: ${ }^{*} P<0.05$. (p) Western blot analysis for PEDF in the eye-cup showed that PEDF expression was significantly reduced 24,36 and $48 \mathrm{~h}$ after LD. Quantification is presented as relative expression normalized against values of tubulin controls to adjust for protein loading. Results are expressed as mean \pm S.D. ( $n=6$ per group). Statistical analyses: one-way ANOVA and Tukey's post hoc test: ${ }^{*} P<0.05$ and ${ }^{* *} P<0.01$

levels of VEGF and PEDF at different times in the neural retina and in the layers corresponding to the RPE/ choriocapillaris/sclera (referred below as the eye-cup). In physiologic conditions, VEGF was 2.1-fold more concentrated in the eye-cup than in the neural retina; however, this ratio was inverted 24-h post LD and seemed to slowly return to normal at 36 and 48-h post LD (Figure 20). Oppositely, PEDF is normally present in the eye-cup, but its concentration is markedly decreased after LD at the same time points (Figure 2p). This suggests that the disruption of the outer BRB that we observed in the LD model could be triggered by an increase in several signals inducing a vasopermeabilty at the apical pole of the RPE.
Activation of the VEGFR-2/Src pathway during RPE permeability. The initiation of vascular permeability through VEGFR-2 is Src kinase-dependent. ${ }^{29}$ To determine whether this pathway was activated in the LD model, the amount of phosphorylated-Src was measured using western blot. Using an antibody specific for the phospho-Tyr416-activated form, we observed a marked activation of Src kinase $6 \mathrm{~h}$ after LD in the eye-cup and in the neural retina (Figures $3 a$ and $b$ ). In parallel, PEDF level showed a constant decrease from $24 \mathrm{~h}$ to $48 \mathrm{~h}$ after LD (Figure 2p), probably leading to a relief of its inhibitory action on VEGFR-2. ${ }^{30}$

To characterize whether the effectors - that is, VEGF receptors VEGFR-1 and -2 - were altered after LD, their 

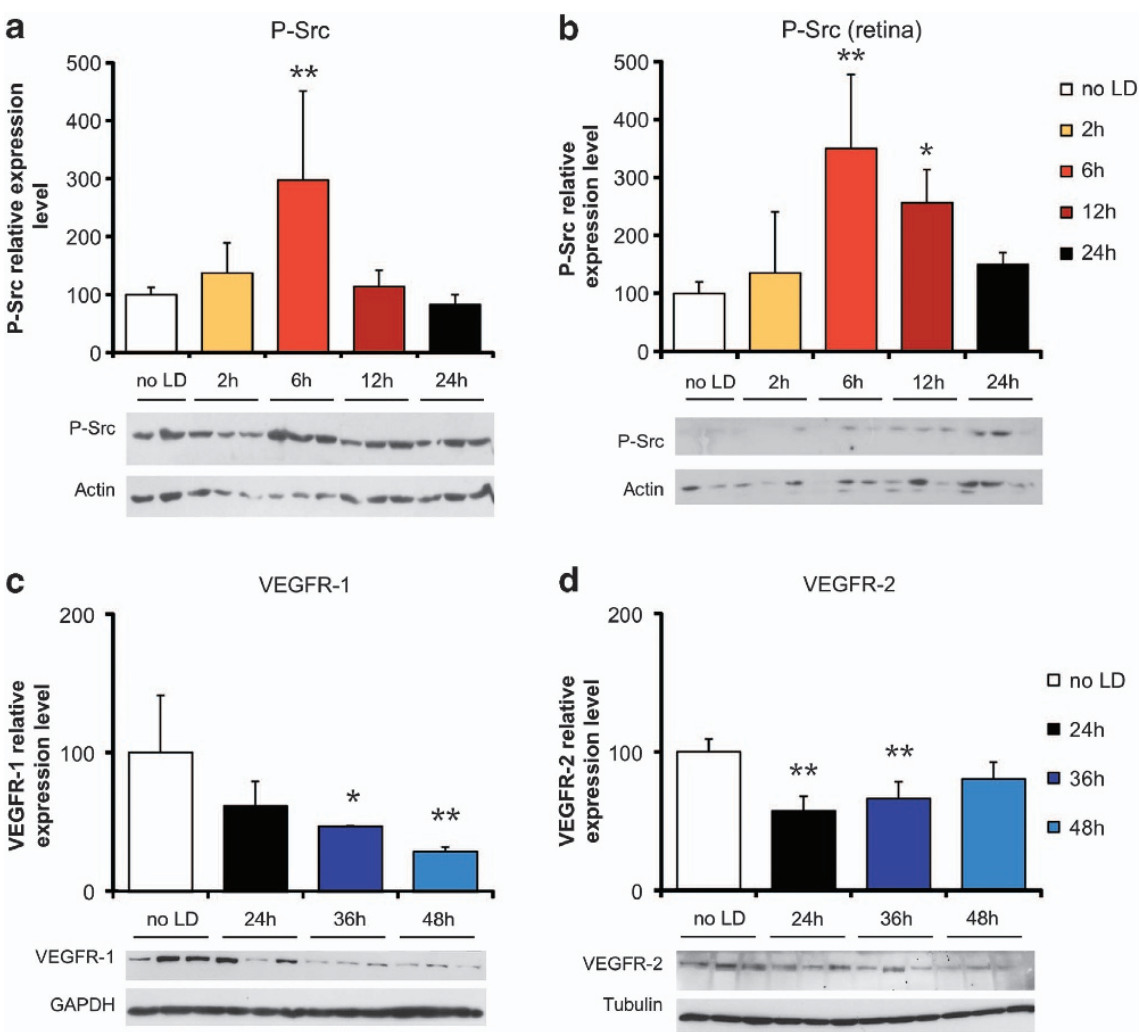

Figure 3 RPE permeability is induced through a VEGFR-2/Src pathway. Western blot analysis showed a three-fold increase in phosphor-Tyr416-Src (P-Src) expression $6 \mathrm{~h}$ after LD in the eye-cup (a), as well as in the neural retina (b). The expression of VEGFR-1 decreased significantly and progressively down to $28 \%$ of basal level at $48 \mathrm{~h}$ after LD (c). At 24 and $36 \mathrm{~h}$ after LD, VEGFR-2 expression was decreased by 45 and 35\%, respectively, and seemed to return to basal level at $48 \mathrm{~h}$ (19\% decrease, not significant - d). Quantification results are expressed as percentage of the control group. Protein expression level was normalized against values of tubulin, GAPDH or actin controls to adjust for protein loading. Results are expressed as mean \pm S.D. ( $n=6$ per group). Statistical analyses: one-way ANOVA followed by Bonferoni's post hoc test: ${ }^{*} P<0.05$ and ${ }^{* *} P<0.01$ (no LD: control group unexposed to light)

expression was quantified in the eye-cup using western blot at different time points. Between 24 and $48 \mathrm{~h}$ after LD, VEGFR-1 level decreased constantly (Figure 3c), whereas VEGFR2 level appeared to come back to its initial level after a decrease in protein level of about $50 \%$ (Figure 3d). These variations further suggested that in the LD model, the RPE permeability was triggered by VEGF activation of a VEGFR-2-dependent pathway at the apical side of the RPE layer (presence of VEGFR-2 and decrease of VEGFR-1 rendering VEGF more available).

VEGF binding on VEGFR-2 is also known to trigger the activation of other intracellular pathways. In consequence, we explored whether different VEGF-R pathways were perturbed after LD or only the Src pathway was transiently activated. For example, in the ARPE-19 cell line under oxidative stress, VEGFR-2 activation leads to Akt phosphorylation, which in turn provides protection to the cells. ${ }^{31}$ On the other hand, VEGFR-2 activation can also lead to cell death, as it has been shown in endothelial cells submitted to peroxynitrite-induced oxidative stress. ${ }^{32}$ In this case, nitration of PI3 kinase switches off the VEGF pro-survival pathway and activates the p38 MAP kinase pro-apoptotic pathway. We thus analyzed whether these pathways could be activated or inhibited in the LD model using western blot analysis of Akt and p38 MAP kinase in the neural retina and in the eye-cup. Neither the protein level nor the phosphorylation state of these proteins was modified in the retina and the RPE during the first $48 \mathrm{~h}$ following LD (Supplementary Figures S1D-O), indicating that the VEGFinduced cell death pathway was activated neither in the photoreceptors nor in the RPE cells. All these results strongly suggest that the RPE permeability occurring after LD is mediated by a VEGF increase leading to Src kinase activation and that this mechanism indirectly induces cell death in the photoreceptor layer knowing that some plasmatic proteins are toxic for neurons, such as albumin. ${ }^{33,34}$

Blocking VEGF in the LD model inhibits RPE permeability and extravascular leakage. To further confirm the involvement of VEGF in the LD model, we constructed a lentiviral vector encoding the cDNA of V65 (LV-V65) - a single-chain antibody directed against VEGF and capable of blocking its activity in different experimental paradigms. ${ }^{35,36}$ We inserted V65 cDNA downstream of the ubiquitous promoter EFS (elongation factor1-alpha short promoter) and added an IRES GFP sequence to the construct to detect transduced cells in vivo. As control, a lentiviral vector encoding GFP only was used (Supplementary Figure S2A). Firstly, we assessed that the lentiviral construct was able to express and secrete V65 from transduced cells in vitro and in vivo (Supplementary Figures S2B-E). Secondly, to 
demonstrate that the LV-V65 vector was able to inhibit VEGF biological activity in vivo, we used laser-induced CNV, as the role of VEGF is widely accepted in this model ${ }^{37}$ and a marked inhibition of neovascularization has been observed. (Supplementary Figures S2F-J). This demonstrated that the LV-V65 vector was able to abrogate VEGF biological activity in vivo.

We thus tested the effect of VEGF inhibition by V65 gene transfer in the LD model. Mice received a subretinal injection of LV-V65 and were subjected to LD 4 weeks later. In the control groups (no treatment prior to LD, sham- or LV-GFPinjected), beta-catenin staining of flat-mounted RPE showed a clear translocation toward the cytoplasm of RPE cells (Figures $4 b-d)$. LV-V65 treatment dramatically reduced the translocation of beta-catenin. Although light staining was found in the cytoplasm of RPE cells, the protein was retained in the periphery of the cells to form a continuous distribution at the interface of the cells (Figures $4 \mathrm{e}$ and $\mathrm{f}$ ), which is in line with maintenance of outer BRB integrity. In the control LD groups, beta-catenin was translocated into the cytoplasm and was fully absent from the cell junction region, forming a 'negative image' of the normal situation.

To reveal potential BRB breakdown after LD, we studied extravascular plasma leakage by immunostaining of the serum albumin on cryostat sections of the retina in the different groups. Results analyzed 10 days after LD revealed an important plasma leakage occurring mainly from the choriocapillaris and reaching the inner side of the retina in the sham- and LV-GFP-injected groups. Concomitantly, a significant thinning of the ONL occurred (see DAPI staining in Figures $4 \mathrm{k}-\mathrm{p}$ ) compared with the non-exposed retina (Figures $4 \mathrm{i}$ and $\mathrm{j}$ ). However, in the LV-V65-treated group, only discrete albumin leakage confined to the outermost retina was observed, suggesting an almost complete suppression of hyperpermeability (Figures $4 q$ and $r$ ). Moreover, retinal morphology appeared much better preserved in mice expressing V65. This collective evidence confirmed that antagonizing VEGF in the LD model significantly reduced RPE hyperpermeability and thus the extravascular plasma leakage from the choriocapillaris toward the neural retina.

Blocking VEGF in the LD model is neuroprotective for photoreceptors. As we hypothesized that RPE permeability was involved in the photoreceptor degeneration occurring after LD, we next evaluated 10 days after LD whether VEGF blockade by the V65 gene transfer was also beneficial to photoreceptors by blocking RPE permeability. Indeed, at this time, the damage induced at the level of the layer of photoreceptors peaks in effect. ${ }^{13}$ Using the same aforementioned regimen, adult Balb/c mice were treated by a subretinal injection of LV-V65 or a control treatment (LV-GFP or Sham injection) 4 weeks before LD. Then, 10 days after $L D$, the electroretinogram response (ERG) was quantified in scotopic conditions. Compared with unlesioned animals, the a-wave, reflecting the activity of photoreceptors, was almost completely abolished in sham-treated animals or in animals that received no treatment prior to LD. In contrast, a significant protection of the a-wave in the LV-V65 group was observed, whereas animals that received the LV-GFP control vector showed an intermediate response (Figures $5 \mathrm{a}$ and b). Quantification of the b-wave amplitude, reflecting the activity of second-order neurons, led to the same conclusions (Figures $5 \mathrm{a}-\mathrm{c}$ ). Following ERG measurement, we estimated transgene expression level using quantitative PCR on eyecup samples. In the LV-V65-treated group, the level of V65 expression correlated significantly with a- and b-wave maximal amplitudes (Figures $5 d$ and e). In the LV-GFP control group, there was no correlation between transgene expression level and ERG amplitudes. This result confirms that blocking RPE hyperpermeability by V65 gene transfer markedly protected photoreceptor function, although the vector itself may have some unspecific effect. To analyze whether this trophic effect observed at the functional level reflected a better survival of photoreceptors, we treated another series of animals with the same protocol and assessed photoreceptor survival by the measurement of ONL thickness. In accordance with ERG results, we observed a massive thinning of the ONL in control groups. Photoreceptor loss was negligible in the LV-V65-treated group, whereas animals treated with the control vector showed an intermediate loss (Supplementary Figure S3). Interestingly, photoreceptor protection after V65 gene transfer was homogenous across the dorsoventral axis of the retina, suggesting that diffusion of the V65 protein brought its trophic effect outside the transduced area of the retina (Supplementary Figure S3).

We further characterized the morphology of rod and cone OS using Peripherin-RDS and SWL-opsin as markers (Figure 6). In sham-treated animals or in animals that received no treatment prior to $L D$, both markers were totally abolished, reflecting the dramatic photoreceptor degeneration (Figures 6b, c, g and h). In LV-GFP-treated animals, Peripherin-RDS staining was still present but OS appeared shorter in length and disorganized (Figure 6d), whereas the expression in LV-V65-treated animals OS appeared well organized and with appropriate length (Figure 6e) compared with the unlesioned retinas (Figure 6a). Similar results were found for the cone OS marker SWL-opsin except that in the LV-GFP group, in addition to a weaker expression level compared with the unlesioned animals and LV-V65-treated animals, the protein was partly mislocalized to the axon and cone pedicle (Figures $6 f-j$ ).

To demonstrate that not only photoreceptors were protected by $\mathrm{V} 65$ gene transfer but also that the retinal circuitry was preserved and was able to convey an interpretable signal to the brain, we studied the optokinetic reflex $(\mathrm{OR})$ in the different groups. This test is able to discriminate rod and cone functions. ${ }^{38}$ In the case of the $\mathrm{Balb} / \mathrm{c}$ used in the present study, the scotopic OR was significantly higher than the photopic one (Supplementary Figure S4). We thus measured the scotopic OR in the different groups before gene transfer and light exposure, 2 weeks after gene transfer to evaluate the effect of the subretinal injection on OR, and 10 days after LD to assess the effect of light exposure. In all the control groups (control vector-treated animals, sham-treated animals or animals that received no treatment prior to LD), we detected a drop in OR performance after LD corresponding to the photoreceptor cell death (Figures $7 a-c)$. On the 

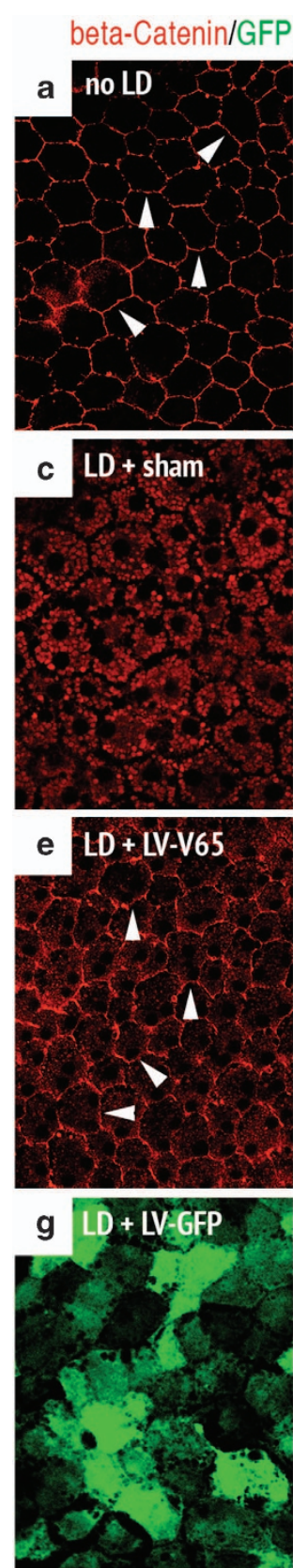
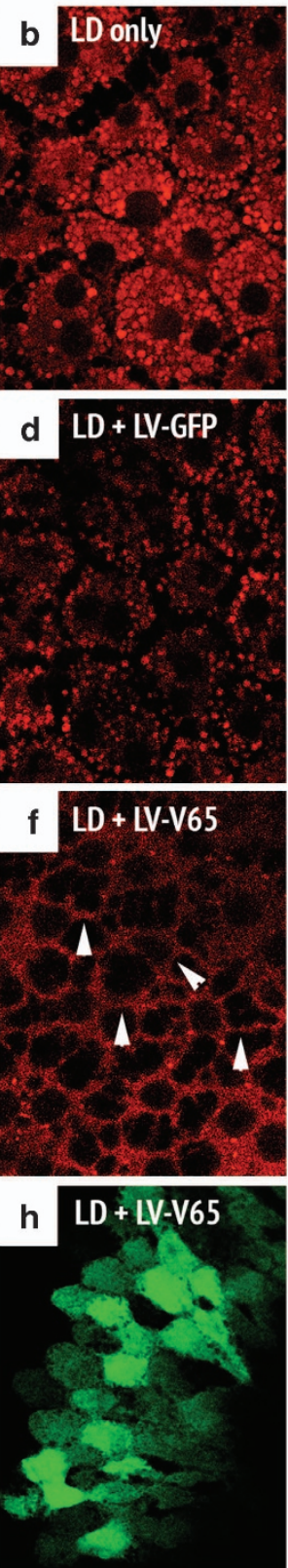

\section{Albumin/DAPI}
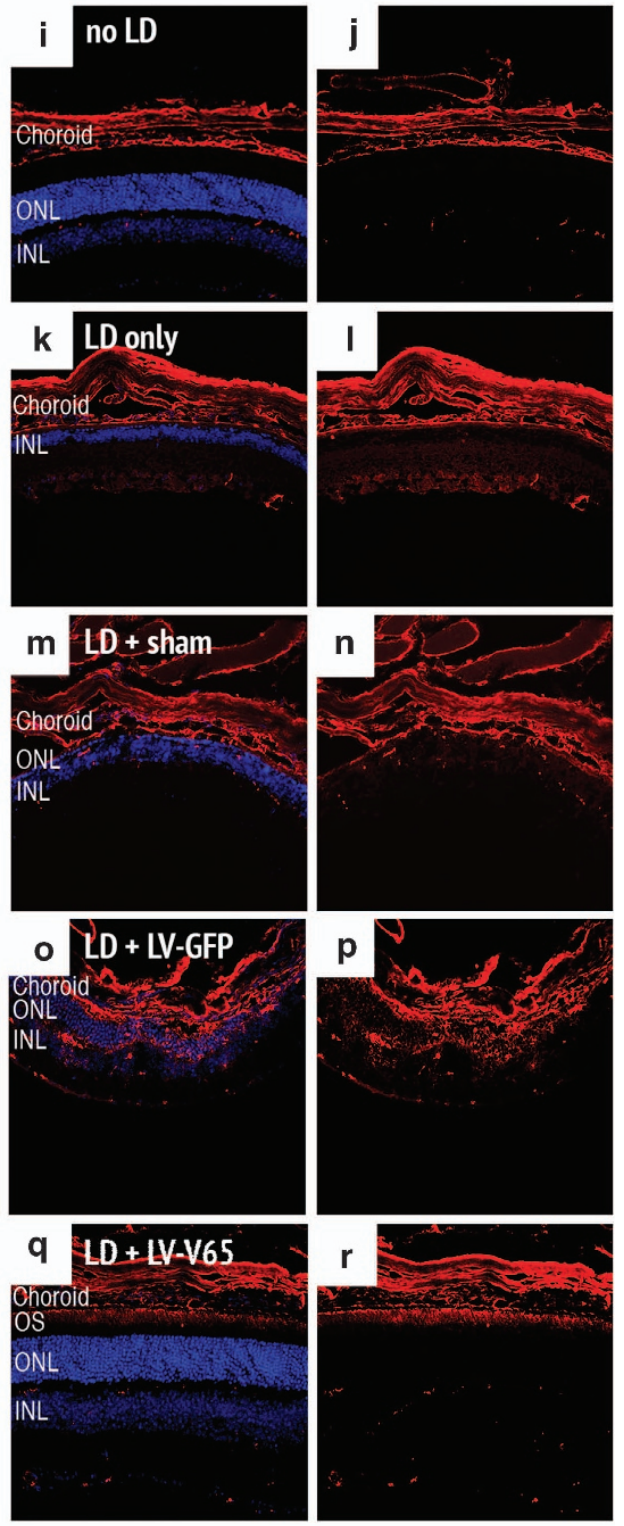

Figure 4 Blocking VEGF in the LD model inhibits RPE permeability and extravascular albumin leakage. The left panel shows the expression of beta-catenin stained on flat-mounted RPE and analyzed on control unexposed to light (a) or $24 \mathrm{~h}$ after LD in the following groups: no treatment prior to LD (b), Sham- (c), LV-GFP- (d) and LV-V65treated animals (e and f). In unexposed control, the interface of cells is clearly delineated by beta-catenin staining (white arrowheads in a). In the control groups (no treatment prior to LD, sham- and LV-GFP-injected), beta-catenin staining shows a clear translocation of the adherens-junction component toward the cytoplasm of RPE cells and is absent at the cell contacts. Contrariwise, in the LV-V65-treated group, beta-catenin staining is maintained at the interface of cells (white arrowheads in $\mathbf{e}$ and $\mathbf{f}$ ), although slightly more diffuse than in absence of exposure to light. Note that GFP expression of LV-GFP $(\mathbf{g})$ and LV-V65 (h) vectors are observed at the RPE level ( $n=5$ per group magnification $\times 400$ (confocal analysis) - red: beta-catenin and green: GFP). The right panel presents serum albumin staining of mice unexposed to light (i and $\mathrm{j}$ ), untreated before LD (k and I), Sham- ( $\mathbf{m}$ and $\mathbf{n})$, LV-GFP- ( $\mathbf{0}$ and $\mathbf{p})$ and LV-V65 ( $\mathbf{q}$ and $\mathbf{r})$-treated groups and analyzed 10 days after LD. Plasma leakage is observed in Sham- and LVGFP-injected groups. Albumin positivity is evident in the choriocapillaris, RPE, ONL, OPL, INL, IPL and GCL. However, in the LV-V65-treated group, albumin staining is only limited to the outer segments of the photoreceptors, which appeared to be protected by the blockade of VEGF $(n=8$ per group - magnification $\times 400$, scale bar: $100 \mu \mathrm{m}-$ red: albumin and blue: Dapi, OS: outer segments, ONL: outer nuclear layer, INL: inner nuclear layer)

contrary, OR performance was preserved after LD in the LV-V65-treated group (Figure 7d), demonstrating that the retinal circuitry mediating OR response was fully functional in this group.

\section{Discussion}

We demonstrated that transient stress of the RPE might have irreversible consequences on photoreceptor integrity 


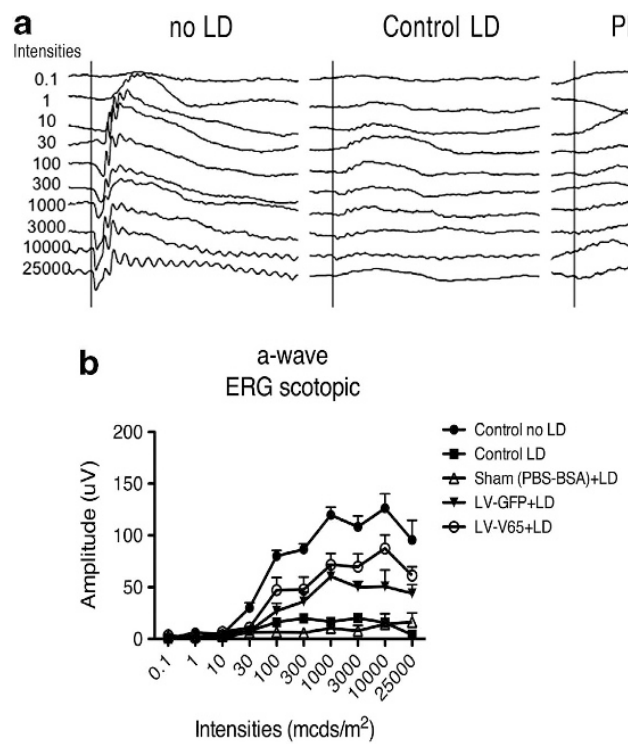

PBS-BSA+LD

LV-GFP+LD

LV-V65+LD
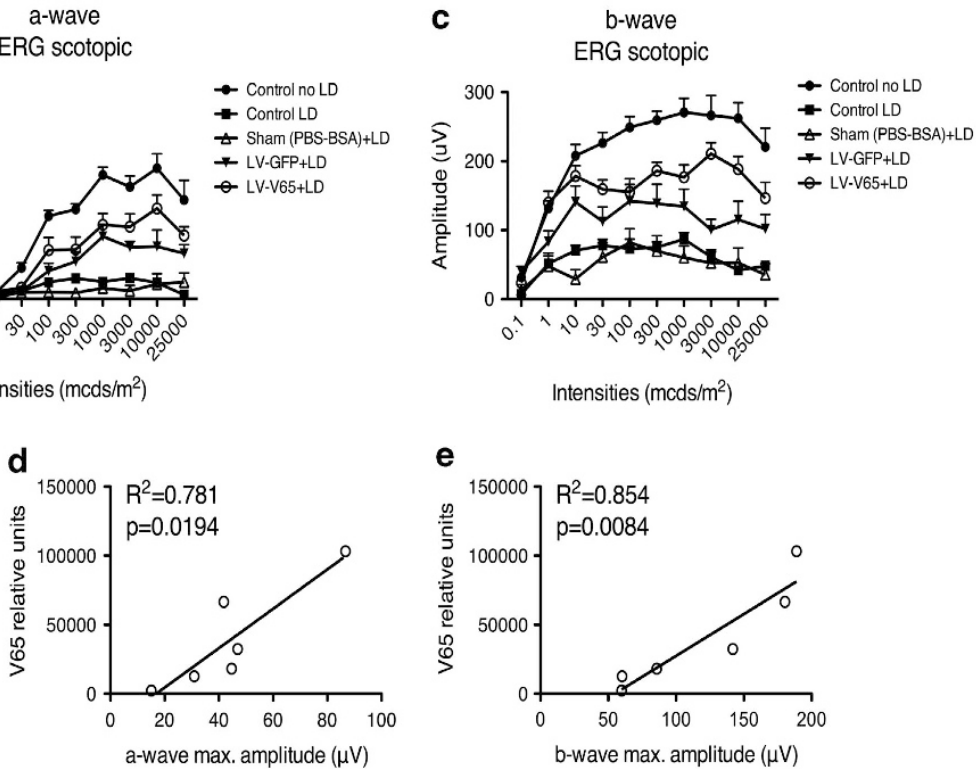

Figure 5 Blocking VEGF in the LD model is neuroprotective for photoreceptors. (a) Representative scotopic electroretinogram response for both the a- and b-wave amplitudes. The quantification of the a-wave (b) amplitude at the different stimuli intensities reveals that response of the LV-V65-treated group is intermediate between control unexposed to light and LV-GFP-treated mice responses. Note that LV-V65-treated mice response is significantly higher than LV-GFP-treated mice response for the following intensities: $3 \times 10^{3}(P<0.05), 10 \times 10^{3}(P<0.01)$ and $25 \times 10^{3}(P<0.05) \mathrm{mcds} / \mathrm{m} 2$. Similarly, the quantification of the b-wave amplitude at the different intensities stimuli showed the same result (c). LV-V65-treated mice response was significantly higher compared with LV-GFP-treated mice response for the same intensities: $3 \times 10^{3}(P<0.01)$, $10 \times 10^{3}(P<0.05)$ and $25 \times 10^{3}(P<0.05) \mathrm{mcds} / \mathrm{m} 2$. Results are expressed as mean \pm S.E. $(n=8$ per group); statistical analyses: two-way ANOVA and Bonferroni's post hoc test. This experiment was reproduced with different batches of lentiviral vectors and led to the same results. Transgene expression level quantified by quantitative PCR revealed that functional rescue of photoreceptor correlates with the level of $\mathrm{V} 65$ expression for both the a-wave (d) and the b-wave (e) maximal amplitudes ( $n=10$ per group)

and survival. Indeed, exposure to intense visible light triggers outer BRB breakdown by activation of VEGF signaling. The resulting hyperpermeability appears to be involved in lightinduced photoreceptor apoptosis. Moreover, outer BRB breakdown and subsequently photoreceptor apoptosis can be prevented by VEGF inhibition.

During the course of light damage, VEGF level is downregulated in the eye-cup (RPE, choroid) and upregulated in the neural retina, indicating a loss of polarity of trophic factor secretion by RPE cells after LD (Figure 2). This reversion of VEGF distribution is associated with activation of the VEGFR2/Src pathway in the eye-cup presumably at the apical side of the RPE (Figure 3), showing an abnormal polarity of VEGF diffusion. As a consequence, there is a hyperpermeability of RPE cells and a subsequent major contribution to the degeneration of photoreceptors. In line with our results, isolated human RPE cells have recently been shown to respond to white light by increased expression and secretion of VEGF. The effect of light could be inhibited by interference with VEGF-R signaling. ${ }^{39}$ Another recently published article showed that basolateral VEGF secretion from RPE cells is indirectly triggered by light through phagocytosis of the shed OS. Contrariwise to the experiments described here, the light stimulus used by Ueta et al. ${ }^{40}$ remained in a physiological range - that is, 5-15lux on pigmented mice - probably explaining the fact that they observe VEGF secretion at the basolateral side only. In our case, the system might be overwhelmed because of toxic light stimulation - 5000 lux in albino mice - leading to VEGF secretion at the apical side. We demonstrate that the toxicity of VEGF on photoreceptors is not a direct action on these cells but rather secondary to VEGF-induced RPE permeability. This rupture of the epithelium integrity likely leads to changes of metabolite exchange between the retina and the choroid that may further destabilize photoreceptors after exposure to high levels of light. Indeed, a massive influx of $\mathrm{O}_{2}$ and nutrients through over-permeability of the RPE may increase oxidative stress in photoreceptors and ultimately lead to neuronal death. This can be paralleled to what occurs after ischemia/reperfusion injury in other organs, which leads to a high level of oxidative stress and subsequent cellular damages as shown, for example, in the liver ${ }^{41}$ or in the kidney. ${ }^{42}$ Another possibility 
Peripherin-RDS/DAPI
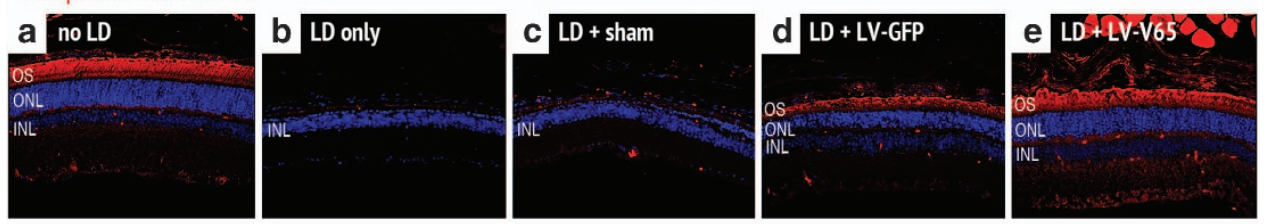

SWL-opsin/DAPI
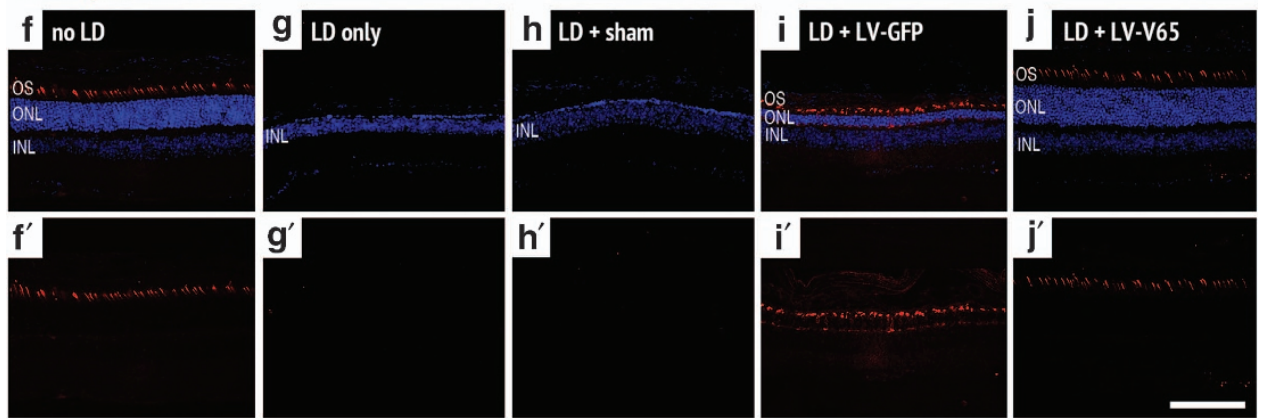

Figure 6 Blocking VEGF in the LD model preserved the rod and cone outer segment morphology. Peripherin-RDS expression, normally localized in rod and cone OS of control unexposed to light (a), is completely abolished 10 days after light exposure (b). Peripherin-RDS expression is also absent in the sham-treated retina (c). Although Peripherin-RDS staining is observed in both LV-GFP- (d) and LV-V65 (e)-treated retinas, the OS in the LV-GFP-treated group appeared shorter and disorganized compared with those of LV-V65-treated group. In the LV-V65-treated group, the OS remains well organized and has an appropriate length. Similar result was obtained for the cone OS marker SWL-opsin analyzed 10 days after LD. SWL-opsin staining, normally found in the cone OS of control unexposed to light (f and $f^{\prime}$ ), is completely abolished after light exposure $\left(\mathbf{g}\right.$ and $\left.\mathbf{g}^{\prime}\right)$. Similarly, the expression of SWL-opsin is absent in sham-treated retina ( $\mathbf{h}$ and $\left.\mathbf{h}^{\prime}\right)$. SWL-opsin expression is maintained in LV-GFP- (i and $\left.\mathbf{i}^{\prime}\right)$ and LV-V65(j and $\mathbf{j}^{\prime}$ )-treated groups; however, in addition to a weaker expression in the LV-GFP-treated group, the protein was partly mislocalized to the axon and cone pedicle (i') $(n=8$ per group - magnification $\times 400$, scale bar: $100 \mu \mathrm{m}$ - red: Peripherin/SWL-opsin and blue: Dapi)

a

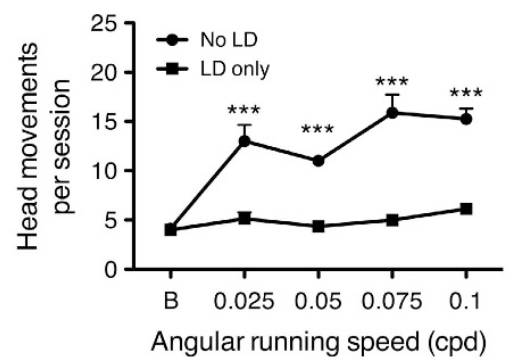

b OR: Sham (PBS-BSA)+LD Group

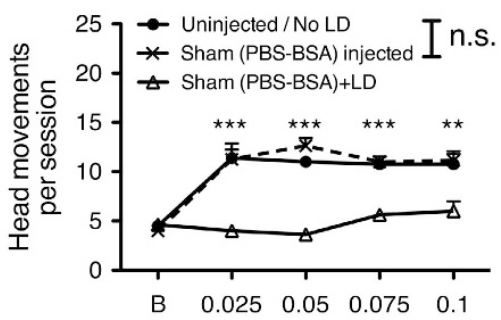

Angular running speed (cpd)
C

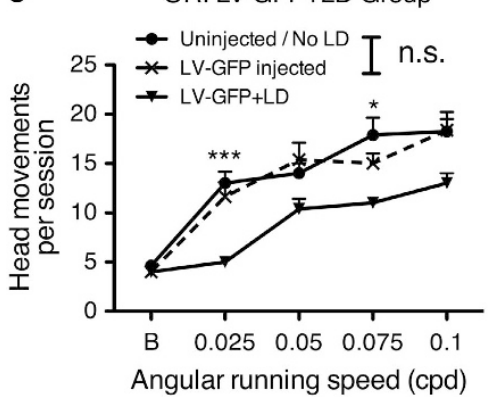

d

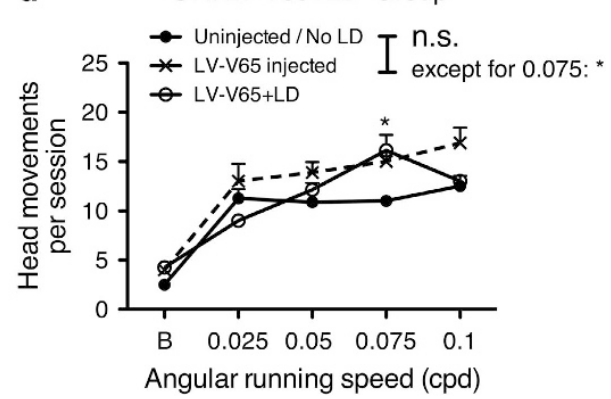

Figure 7 Blocking VEGF in the LD model preserved visual acuity. The optokinetic response (OR) was quantified in the following experimental groups: no treatment prior to LD (a), Sham- (b), LV-GFP- (c) and LV-V65 (d)-treated groups. The quantification of the OR was measured at the beginning of the experiment (prior the injection and LD), 2 weeks after subretinal injection and 10 days after LD. Statistical analysis presented in the figures compared OR before and after light exposures. Results show that the subretinal injection did not affect OR in the different injected groups (b-d), except in the LV-V65-treated group for 0.075 cpd. Contrariwise, 10 days after light exposure, ORs recorded in the control groups (no treatment prior to LD and Sham-injected) were significantly impaired and no difference was observed compared with background value. OR was still present but decreased in the LV-GFP-treated group after LD. Blocking VEGF preserved OR after light exposure in the LV-V65-treated group. Results are expressed as mean \pm S.E. Statistical analyses: one-way ANOVA and Tukey's post hoc test: $n . s .:$ nonsignificant, ${ }^{*} P<0.05,{ }^{* \star} P<0.01$ and ${ }^{* * *} P<0.001$ ( $n=8$ per group) 
strongly suggested by our observation is that, similarly to what happens in different stroke models, direct contact between plasmatic proteins and photoreceptors is detrimental to the latter. Indeed, several publications have shown that the sole presence of albumin is toxic for neurons. ${ }^{33,34}$ Finally, one can also postulate that following direct damage induced by exposure to toxic levels of white light, the RPE cells are no longer able to ensure photoreceptor homeostasis. ${ }^{43,44}$ In the present LD paradigm, the toxicity of VEGF is furthermore reinforced by several parameters. Firstly, RPE permeability might amplify VEGF leakage to the apical side of RPE cells by the deregulation of VEGF secretion, as was shown recently by Kunchithapautham and Rohrer ${ }^{45}$ on the RPE cell line ARPE19 exposed to sublytic levels of membrane-attack complex. VEGF secretion at the apical side of RPE cells might thus be auto-inducible, leading to a vicious cycle. Secondly, in our model, PEDF is downregulated, thus making VEGF more active for VEGFR-2, whose activation leads to RPE permeability. ${ }^{9,30}$

In order to evaluate a possible direct VEGF action on photoreceptor death, we measured phosphorylated p38, which is known to trigger apoptosis in stressed cells, ${ }^{32}$ and we found no differences between groups nor a potential survival stimulation by VEGF through Akt phosphorylation, suggesting that VEGF has no direct effect on photoreceptor cells in this context. Several studies have revealed different pathways, such as c-fos and AP-1, involved in photoreceptor death. ${ }^{46,47}$ Interestingly, LD under high-light exposure does not depend on transducin, whereas light toxicity through lowlight exposure requires transducin. ${ }^{48}$ It would be interesting to study these different pathways when VEGF is inhibited to understand how the indirect effect of the BRB breakdown contributes to photoreceptor death. Indeed, an edema does not usually produce a rapid degeneration of the photoreceptors, suggesting that the light damage has severely affected the survival/death balance of these cells and that RPE leakage ultimately pushes the balance toward the death pathway. Such study would help to better delineate the relative contribution of the direct and the indirect inductions of cell death.

In our study, an acute strong stress produces a transient, but deleterious, VEGF action, suggesting that in certain diseases an acute stress can start to change RPE environment and then, on the long term, provide a toxic milieu. With age, oxidative stress of photoreceptors brings deposits at the level of Bruch's membrane; ${ }^{12}$ deregulation of the complement pathway leads to RPE dysfunction, at least in vitro, ${ }^{45}$ by enhancing VEGF secretion; and myeloid cells can enhance, in certain cases, the side effects derived from this environment. ${ }^{49,50}$ It would be important to study the sequence of these events in regards to RPE dysfunction to identify the key factors before a vicious cycle that maintains elevated VEGF levels is established.

Anti-VEGF treatment is currently and efficiently used to treat vascular permeability in wet-AMD ${ }^{51}$ and to decrease subretinal edema. ${ }^{52,53}$ This recovery was often mainly attributed to the vascular effect of the anti-VEGF treatment. However, in view of our results, we cannot exclude that in certain cases, the amelioration may also be because of a re-establishment of the RPE integrity preventing inward fluid flux from the choroid. A similar statement can be hypothesized for macular edema occurring during diabetic retinopathy depending on VEGF levels and distribution. Our results strongly suggest that it is also important to investigate RPE integrity in these diseases to ameliorate diagnosis and therapeutic intervention.

\section{Materials and Methods}

Animals. Animals were handled in accordance with the 'Animals in Research Committee' statement of the Association for Research in Vision and Ophthalmology (Rockville, MD, USA) and approved protocols of the local institutional committee (Office des Affaires Vétérinaires du Canton de Vaud, Lausanne, Switzerland). Mice were housed in communal cages (five to six animals per cage), maintained in controlled temperature $\left(22^{\circ} \mathrm{C}\right)$ and light $(12 \mathrm{~h}$ light-dark cycle) and fed ad libitum. This work included wild-type C57/BI6 mice (provided by Charles River, Lyon, France) and Balb/c mice (provided by Harlan Laboratories BV, Venray, Netherlands). All experiments were run with 8-12 weeks old male mice.

Light damage and degeneration. Photic injury to mouse retinas was carried out as described by Wenzel et al. ${ }^{54} \mathrm{Balb} / \mathrm{c}$ mice that display a susceptibility to LD were kept for 4 weeks in cyclic light at or below 80 lux. Mice were dark-adapted overnight (16 h) and then immediately exposed to 5000 lux of diffuse white-fluorescent light (TLD36 W/965 tubes, Philips, Hamburg, Germany, UV-impermeable diffuser) for $1 \mathrm{~h}$ in a cage with reflective interior. Fluorescentwhite light emitted from TLD36 W/965 tubes has an emission spectrum similar to daylight to mimic excessive exposure (reviewed in Wenzel et $a l^{13}{ }^{13}$ ). After exposure, mice returned to complete darkness until retinal morphology was analyzed or until their eyes were prepared for western blotting (between $2 \mathrm{~h}$ and 10 days). Mice were then killed by cervical dislocation and decapitation under dim red light conditions. To evaluate the degeneration, measures of the ONL thickness were taken at 300 and $400 \mu \mathrm{m}$ dorsally and ventrally, respectively, from the optic nerve head on cryosections, and data were pooled as a mean for each points to evaluate degenerating central retina. The measures were taken using an Olympus BX60 microscope equipped for epifluorescence (Olympus Suisse SA, Aigle, Switzerland) coupled to the Cell-P software (Soft Imaging System, Olympus, Hamburg, Germany).

Protein extraction and western blotting. Neural retinas and eye-cups were collected in $200 \mu$ lo homogenization buffer composed of $137 \mathrm{mM} \mathrm{NaCl}, 20 \mathrm{mM}$ Tris pH 8, 1\% Nonidet-P40, 10\% Glycerol, 2 mM EDTA, 1/100 phosphatase inhibitors 1 and 2 (Sigma, Steinlheim, Germany) and complete Mini ESTA-free anti-protease (Roche Diagnostics, Indianapolis, IN, USA). The protein content was determined using a Bradford assay (Bio-Rad Laboratories, Munich, Germany).

Western blotting: Proteins were loaded and separated using SDS-PAGE and blotted onto PVDF membranes. After blocking in Tris-buffered saline containing $0.1 \%$ Tween-20 (TBS-T) and 5\% dry-milk, membranes were incubated with primary antibodies overnight at $4{ }^{\circ} \mathrm{C}$ in TBS-T containing $5 \%$ milk or $5 \%$ BSA. Primary antibody providers and concentrations are described in Supplementary Table 2. After incubation with the appropriate peroxydase-coupled secondary antibodies in blocking buffer for $1 \mathrm{~h}$, signal was revealed by a chemiluminescent substrate (ECL plus western blotting detection system, GE Healthcare, Buckinghamshire, UK), and then membranes were exposed to hyperfilm ECL (Hyperfilmchemiluminescence film, GE Healthcare). Results were analyzed using the ImageJ software (National Institutes of Health, Bethesda, MD, USA) to determine relative expression.

VEGF content and cell-death quantification. VEGF content was evaluated on neural retinas and eye-cups homogenized as previously mentioned. VEGF ELISA was performed using the VEGF Quantikine Immunoassay (R\&D systems, Minneapolis, USA) following the manufacturer's instructions. Cell death was quantified on retinas collected 24, 36 and $48 \mathrm{~h}$ after LD using Cell Death Detection Kit (Roche Diagnostics) according to the manufacturer's recommendations. In this case, retinas were homogenized directly with CDD buffer included in the kit. The timepoint chosen corresponds to the peak of apoptosis (reviewed in Wenzel et $a l^{13}{ }^{13}$. Results were obtained using the Revelation software connected to a plate reader MRXII (Dynex technologies, Chantilly, VA, USA). 
Lentiviral vectors and in vivo gene transfer. Lentiviral constructs, generation of lentiviral particles and in vivo delivery are described in details in Supplementary Methods. Briefly, lentiviral vectors used in this study are selfinactivating, HIV-1-derived vectors. Lentiviral particles were produced by transient co-transfection of 293T cells with a lentiviral shuttle plasmid, a packaging plasmid and an expression plasmid encoding the heterologous envelope VSV-G. Lentiviral particles were harvested $48 \mathrm{~h}$ after transfection in the cell supernatant, concentrated by ultracentrifugation and measured by p24 ELISA. Adult mice were treated by subretinal delivery of a vector dose corresponding to $150 \mathrm{ng}$ of p24 protein. LVs were injected in C57/BI6 mice 5 days before laser injury and subretinal injections were performed 3 or 4 weeks before LD in Balb/c mice.

\section{Histology}

Cryosections: eyes were enucleated immediately after killing of the animals and fixed for $1 \mathrm{~h}$ in $4 \%$ paraformaldehyde (PFA) in phosphate-buffered saline (PBS). After overnight incubation in $30 \%$ sucrose, the eyes were embedded in albumin from hen egg white (Fluka, Buchs, Switzerland) and processed into $14-\mu \mathrm{m}$-thick cryosections.

RPE flat-mount preparations: Enucleated eyes were immediately fixed in 4\% PFA for $20 \mathrm{~min}$ and transferred to PBS. The eyes were sectioned at the equator, and the anterior half including the lens and the vitreous bodies were discarded. Neural retinas were carefully peeled from the eye-cup. The posterior part containing the RPE and choroid was dissected into quarters by four radial cuts and conserved in PBS at $4^{\circ} \mathrm{C}$ until subsequent use.

Immunolabelling: Immunohistochemistry on cryosections was performed as described previously. ${ }^{38,55}$ Briefly, the first antibody was incubated overnight at $4{ }^{\circ} \mathrm{C}$ in PBS containing $0.2 \%$ Triton $\mathrm{X}-100$ and $10 \%$ normal goat serum (NGS) (Dako, Zug, Switzerland). Primary antibody providers and concentrations are described in Supplementary Table 1. When antibodies were not preconjugated directly with the fluorochrome, revelation was carried out with the appropriate Alexa-633 (Molecular Probes, Eugene, OR, USA) or Alexa-488 (Molecular Probes)-coupled secondary antibody and incubated $1 \mathrm{~h}$ at $37^{\circ} \mathrm{C}$. After incubation, slides were washed three times in PBS. Nuclei were counterstained with 4,6-diamidino-2-phenyindole (DAPI) (Molecular Probes) and mounted under coverslips with Mowiol 4-88 Reagent (VWR International AG, Lucerne, Switzerland). Staining was analyzed using a BX60 microscope equipped for epifluorescence and Cell-P software or using a confocal microscope Leica SP5 AOBS (Leica Microsystems CMS, Mannheim, Germany) using the Leica application suite (ALS) software. For immunolabelling on RPE flat mounts, the blocking solution was composed of PBS with $10 \%$ NGS and $1 \%$ Triton X-100. Primary antibody was diluted in the blocking solution and incubated overnight at $4{ }^{\circ} \mathrm{C}$. Primary antibody providers and antibody concentrations are described in Supplementary Table 1. Detection of primary antibodies was carried out with Alexa-633- Alexa-488- or Cy3-coupled secondary antibodies diluted in PBS with $10 \%$ NGS and $0.1 \%$ Triton X-100 and incubated $1 \mathrm{~h}$ at RT. Flat mounts were mounted as cryosections. Staining was analyzed using a Leica SP5 AOBS confocal microscope and ALS software. TUNEL Staining was performed with the Dead End Colorimetric TUNEL System Kit (Promega, Madison, WI, USA).

Electroretinograms. ERG recordings were performed as previously described. ${ }^{55}$ After dark adaptation mice were anesthetized with ketamine $(100 \mathrm{mg} / \mathrm{kg})$ and xylazine $(15 \mathrm{mg} / \mathrm{kg})$, and pupils were dilated by $0.5 \%$ tropicamid (Novartis Pharma, Bern, Switzerland). The mouse was placed in the Ganzfeld stimulator of a Multiliner Vision apparatus (Jaeger/Toennies, Höchberg, Germany). The corneal ERG was then recorded under scotopic conditions in response to single flashes of white light ranging from $1 \times 10^{-4}$ to 25 candelas $\mathrm{cd} \mathrm{s} \mathrm{m}^{-2}$.

Optokinetic Reflex. The experimental setup and the procedure were previously described in details. ${ }^{38}$ Briefly, Balb/c mice were tested in an optokinetic drum with spatial frequencies increasing from 0.025 to 0.1 cycles per degree (cpd), starting with scotopic condition (dim-light inferior to 0.01 lux), followed by photopic condition (650 lux). During each session, the drum rotated alternatively clockwise and anticlockwise, by inverting the rotation direction at mid-session, and behavior was recorded using a camcorder enabling infrared recording in scotopic conditions (Sony CDR-HC90E). Head movements were then scored: clockwise movement drives tracking through the left eye, whereas counterclockwise motion activates the right eye. ${ }^{56} \mathrm{~A}$ movement was counted when the animal followed the drum rotation at the same angular speed and in the same direction for a minimum of $15^{\circ}$. A level of $P<0.05$ (two-tailed) was considered significant. The OR of Balb/c mice not exposed to LD was first evaluated in both photopic and scotopic conditions. Then, the OR response was evaluated in Balb/c mice submitted to LD. In this case, the OR was only analyzed under scotopic conditions at three different time points: at the beginning of the experiment, after the subretinal injections (LV-GFP, LV-V65 or Sham) in order to evaluate the effect of the injection and finally, 10 days after LD.

Statistical analysis. All data were analyzed using Prism 5.0 Software (GraphPad Software Inc., San Diego, CA, USA). The different statistical analyses are mentioned in each figure legend. A level of $P<0.05$ (two-tailed) was considered significant.

\section{Conflict of Interest}

The authors declare no conflict of interest.

Acknowledgements. We thank Vérène Pignat, Dana Wanner and Meriem Tekaya for their excellent technical supports. This work was supported by "Association Française contre les Myopthies" (AFM), the Provisu Foundation, the Swiss National Science Foundation, the OpenEyes foundation.

\section{Author Contributions}

$M C, A-P B$ and $Y A$ designed the experiments, analyzed data and wrote the paper. $M C$ and A-PB performed the experiments. TA isolated and characterized the V65 single-chain antibody. J-AP performed the laser-induced model of choroidal neovascularization. MS, CG and AW setup the light-damage model and performed resin-embedded histology. SP and CK participated in gene transfer and western blot experiments. All authors revised and approved the manuscript.

1. Lim LS, Mitchell P, Seddon JM, Holz FG, Wong TY. Age-related macular degeneration. Lancet 2012; 379: 1728-1738.

2. Dawson DW, Volpert OV, Gillis $P$, Crawford SE, Xu H, Benedict $W$ et al. Pigment epithelium-derived factor: a potent inhibitor of angiogenesis. Science 1999; 285: 245-248.

3. King GL, Suzuma K. Pigment-epithelium-derived factor-a key coordinator of retinal neuronal and vascular functions. N Engl J Med 2000; 342: 349-351.

4. Adamis AP, Shima DT, Yeo KT, Yeo TK, Brown LF, Berse B et al. Synthesis and secretion of vascular permeability factor/vascular endothelial growth factor by human retinal pigment epithelial cells. Biochem Biophys Res Commun 1993; 193: 631-638.

5. Lopez PF, Sippy BD, Lambert HM, Thach AB, Hinton DR. Transdifferentiated retinal pigment epithelial cells are immunoreactive for vascular endothelial growth factor in surgically excised age-related macular degeneration-related choroidal neovascular membranes. Invest Ophthalmol Vis Sci 1996; 37: 855-868.

6. Witmer AN, Vrensen GF, Van Noorden CJ, Schlingemann RO. Vascular endothelial growth factors and angiogenesis in eye disease. Prog Retin Eye Res 2003; 22: 1-29.

7. Tong JP, Yao YF. Contribution of VEGF and PEDF to choroidal angiogenesis: a need for balanced expressions. Clin Biochem 2006; 39: 267-276.

8. Praidou A, Androudi S, Brazitikos P, Karakiulakis G, Papakonstantinou E, Dimitrakos S. Angiogenic growth factors and their inhibitors in diabetic retinopathy. Curr Diabet Rev 2010; 6: 304-312

9. Ablonczy Z, Crosson CE. VEGF modulation of retinal pigment epithelium resistance. Exp Eye Res 2007; 85: 762-771.

10. Holz FG, Schutt F, Kopitz J, Eldred GE, Kruse FE, Volcker HE et al. Inhibition of lysosomal degradative functions in RPE cells by a retinoid component of lipofuscin. Invest Ophthalmol Vis Sci 1999; 40: 737-743.

11. Malek G, Johnson LV, Mace BE, Saloupis P, Schmechel DE, Rickman DW et al. Apolipoprotein $E$ allele-dependent pathogenesis: a model for age-related retinal degeneration. Proc Natl Acad Sci USA 2005; 102: 11900-11905.

12. Bird AC. Therapeutic targets in age-related macular disease. J Clin Invest 2010; 120: 3033-3041.

13. Wenzel A, Grimm C, Samardzija M, Reme CE. Molecular mechanisms of light-induced photoreceptor apoptosis and neuroprotection for retinal degeneration. Prog Retin Eye Res 2005; 24: 275-306.

14. Imai S, Shimazawa M, Nakanishi T, Tsuruma K, Hara H. Calpain inhibitor protects cells against light-induced retinal degeneration. J Pharmacol Exp Ther 2010; 335: 645-652.

15. Perche $\mathrm{O}$, Doly M, Ranchon-Cole I. Caspase-dependent apoptosis in light-induced retinal degeneration. Invest Ophthalmol Vis Sci 2007; 48: 2753-2759. 
16. Bush RA, Reme CE, Malnoe A. Light damage in the rat retina: the effect of dietary deprivation of N-3 fatty acids on acute structural alterations. Exp Eye Res 1991; 53 $741-752$.

17. Reme CE. The dark side of light: rhodopsin and the silent death of vision the proctor lecture. Invest Ophthalmol Vis Sci 2005; 46: 2671-2682.

18. Rozanowska M, Korytowski W, Rozanowski B, Skumatz C, Boulton ME, Burke JM et al. Photoreactivity of aged human RPE melanosomes: a comparison with lipofuscin. Invest Ophthalmol Vis Sci 2002; 43: 2088-2096.

19. Vaughan DK, Nemke JL, Fliesler SJ, Darrow RM, Organisciak DT. Evidence for a circadian rhythm of susceptibility to retinal light damage. Photochem Photobiol 2002; 75 : 547-553.

20. Organisciak DT, Vaughan DK. Retinal light damage: mechanisms and protection. Prog Retin Eye Res 2010; 29: 113-134.

21. Bressler NM, Munoz B, Maguire MG, Vitale SE, Schein OD, Taylor HR et al. Five-year incidence and disappearance of drusen and retinal pigment epithelial abnormalities. Waterman study. Arch Ophthalmol 1995; 113: 301-308.

22. Zhou J, Jang YP, Kim SR, Sparrow JR. Complement activation by photooxidation products of A2E, a lipofuscin constituent of the retinal pigment epithelium. Proc Natl Acad Sci USA 2006; 103: 16182-16187

23. Zhao C, Yasumura D, Li X Matthes M, Lloyd M, Nielsen G et al. mTOR-mediated dedifferentiation of the retinal pigment epithelium initiates photoreceptor degeneration in mice. J Clin Invest 2011; 121: 369-383.

24. Ban Y, Rizzolo LJ. A culture model of development reveals multiple properties of RPE tight junctions. Mol Vis 1997; 3: 18

25. Kanuga N, Winton HL, Beauchene L, Koman A, Zerbib A, Halford S et al. Characterization of genetically modified human retinal pigment epithelial cells developed for in vitro and transplantation studies. Invest Ophthalmol Vis Sci 2002; 43: 546-555.

26. Kaur C, Foulds WS, Ling EA. Blood-retinal barrier in hypoxic ischaemic conditions: basic concepts, clinical features and management. Prog Retin Eye Res 2008; 27: 622-647.

27. Konari K, Sawada N, Zhong Y, Isomura H, Nakagawa T, Mori M. Development of the blood-retinal barrier in vitro: formation of tight junctions as revealed by occludin and ZO-1 correlates with the barrier function of chick retinal pigment epithelial cells. Exp Eye Res 1995; 61: 99-108

28. Marrs JA, Andersson-Fisone C, Jeong MC, Cohen-Gould L, Zurzolo C, Nabi IR et al. Plasticity in epithelial cell phenotype: modulation by expression of different cadherin cell adhesion molecules. J Cell Biol 1995; 129: 507-519.

29. Eliceiri BP, Paul R, Schwartzberg PL, Hood JD, Leng J, Cheresh DA. Selective requirement for Src kinases during VEGF-induced angiogenesis and vascular permeability Mol Cell 1999; 4: 915-924.

30. Ablonczy Z, Prakasam A, Fant J, Fauq A, Crosson C, Sambamurti K. Pigment epitheliumderived factor maintains retinal pigment epithelium function by inhibiting vascula endothelial growth factor-R2 signaling through gamma-secretase. J Biol Chem 2009; 284 30177-30186

31. Byeon SH, Lee SC, Choi SH, Lee HK, Lee JH, Chu YK et al. Vascular endothelial growth factor as an autocrine survival factor for retinal pigment epithelial cells under oxidative stress via the VEGF-R2/PI3K/Akt. Invest Ophthalmol Vis Sci 2010; 51: 1190-1197.

32. el-Remessy AB, Bartoli M, Platt DH, Fulton D, Caldwell RB. Oxidative stress inactivates VEGF survival signaling in retinal endothelial cells via PI 3-kinase tyrosine nitration. J Cell Sci 2005; 118(Pt 1): 243-252.

33. Hassel B, Iversen EG, Fonnum F. Neurotoxicity of albumin in vivo. Neurosci Lett 1994; 167: 29-32.

34. Tomkins O, Friedman O, Ivens S, Reiffurth C, Major S, Dreier JP et al. Blood-brain barrie disruption results in delayed functional and structural alterations in the rat neocortex. Neurobiol Dis 2007; 25: 367-377.

35. Afanasieva TA, Wittmer M, Vitaliti A, Ajmo M, Neri D, Klemenz R. Single-chain antibody and its derivatives directed against vascular endothelial growth factor: application for antiangiogenic gene therapy. Gene Ther 2003; 10: 1850-1859.

36. Vitaliti A, Wittmer M, Steiner R, Wyder L, Neri D, Klemenz R. Inhibition of tumor angiogenesis by a single-chain antibody directed against vascular endothelial growth factor. Cancer Res 2000; 60: 4311-4314.

37. YiX, Ogata N, Komada M, Yamamoto C, Takahashi K, Omori K et al. Vascular endothelia growth factor expression in choroidal neovascularization in rats. Graefes Arch Clin Exp Ophthalmol 1997; 235: 313-319.
38. Cachafeiro M, Bemelmans AP, Canola K, Pignat V, Crippa SV, Kostic C et al. Remaining rod activity mediates visual behavior in adult Rpe65-/- mice. Invest Ophthalmol Vis Sci 2010; 51: 6835-6842.

39. Kernt M, Neubauer AS, Liegl RG, Hirneiss C, Alge CS, Wolf A et al. Sorafenib prevents human retinal pigment epithelium cells from light-induced overexpression of VEGF, PDGF and PIGF. Br J Ophthalmol 2010; 94: 1533-1539.

40. Ueta T, Inoue T, Yuda K, Furukawa T, Yanagi Y, Tamaki Y. Intense physiological light upregulates vascular endothelial growth factor and enhances choroidal neovascularization via peroxisome proliferator-activated receptor gamma coactivator-1alpha in mice. Arterioscler Thromb Vasc Biol 2012; 32: 1366-1371.

41. Horvath B, Magid L, Mukhopadhyay P, Batkai S, Rajesh M, Park O et al. A new cannabinoid 2 receptor agonist $\mathrm{HU}-910$ attenuates oxidative stress, inflammation, and cell death associated with hepatic ischemia/reperfusion injury. Br J Pharmacol 2011; 165 : 2462-2478.

42. Yuan Q, Hong S, Han S, Zeng L, Liu F, Ding G et al. Preconditioning with physiological levels of ethanol protect kidney against ischemia/reperfusion injury by modulating oxidative stress. PLoS One 2011; 6: e25811.

43. D'Cruz PM, Yasumura D, Weir J, Matthes MT, Abderrahim H, LaVail MM et al. Mutation of the receptor tyrosine kinase gene Mertk in the retinal dystrophic RCS rat. Hum Mol Genet 2000; 9: 645-651.

44. Vollrath D, Feng W, Duncan JL, Yasumura D, D'Cruz PM, Chappelow A et al. Correction of the retinal dystrophy phenotype of the RCS rat by viral gene transfer of Mertk. Proc Nat Acad Sci USA 2001; 98: 12584-12589.

45. Kunchithapautham K, Rohrer B. Sublytic membrane-attack-complex (MAC) activation alters regulated rather than constitutive vascular endothelial growth factor (VEGF) secretion in retinal pigment epithelium monolayers. J Biol Chem 2011; 286: 23717-23724.

46. Hafezi F, Marti A, Grimm C, Wenzel A, Reme CE. Differential DNA binding activities of the transcription factors AP-1 and Oct-1 during light-induced apoptosis of photoreceptors. Vision Res 1999; 39: 2511-2518.

47. Wenzel A, Grimm C, Marti A, Kueng-Hitz N, Hafezi F, Niemeyer G et al. c-fos controls the "private pathway" of light-induced apoptosis of retinal photoreceptors. J Neurosci 2000; 20: $81-88$

48. Hao W, Wenzel A, Obin MS, Chen CK, Brill E, Krasnoperova NV et al. Evidence for two apoptotic pathways in light-induced retinal degeneration. Nat Genet 2002; 32: 254-260.

49. Houssier M, Raoul W, Lavalette S, Keller N, Guillonneau X, Baragatti B et al. CD36 deficiency leads to choroidal involution via COX2 down-regulation in rodents. PLoS Med 2008; 5: e39.

50. Luhmann UF, Lange CA, Robbie S, Munro PM, Cowing JA, Armer HE et al. Differential modulation of retinal degeneration by $\mathrm{Ccl} 2$ and $\mathrm{C} \times 3 \mathrm{cr} 1$ chemokine signalling. PLoS One 2012; 7: e35551.

51. Martin DF, Maguire MG, Ying GS, Grunwald JE, Fine SL, Jaffe GJ. Ranibizumab and bevacizumab for neovascular age-related macular degeneration. N Engl J Med 2011; 364 1897-1908.

52. Lang GE. Diabetic macular edema. Ophthalmologica 2012; 227(Suppl 1): 21-29.

53. Truong A, Wong TY, Khachigian LM. Emerging therapeutic approaches in the management of retinal angiogenesis and edema. J Mol Med (Berl) 2011; 89: 343-361.

54. Grimm C Wenzel A, Hafezi F, Yu S, Redmond TM, Reme CE Protection of Rpe65-deficient mice identifies rhodopsin as a mediator of light-induced retinal degeneration. Nat Genet 2000; 25: 63-66.

55. Bemelmans AP, Kostic C, Crippa SV, Hauswirth WW, Lem J, Munier FL et al. Lentivira gene transfer of RPE65 rescues survival and function of cones in a mouse model of Leber congenital amaurosis. PLoS Med 2006; 3: e347.

56. Douglas RM, Alam NM, Silver BD, McGill TJ, Tschetter WW, Prusky GT. Independent visual threshold measurements in the two eyes of freely moving rats and mice using a virtual-reality optokinetic system. Vis Neurosci 2005; 22: 677-684.

(1) $\circledast$ C Cell Death and Disease is an open-access journal published by Nature Publishing Group. This work is licensed under a Creative Commons Attribution-NonCommercialNoDerivs 3.0 Unported License. To view a copy of this license, visit http://creativecommons.org/licenses/by-nc-nd/3.0/

\section{Supplementary Information accompanies this paper on Cell Death and Disease website (http://www.nature.com/cddis)}

